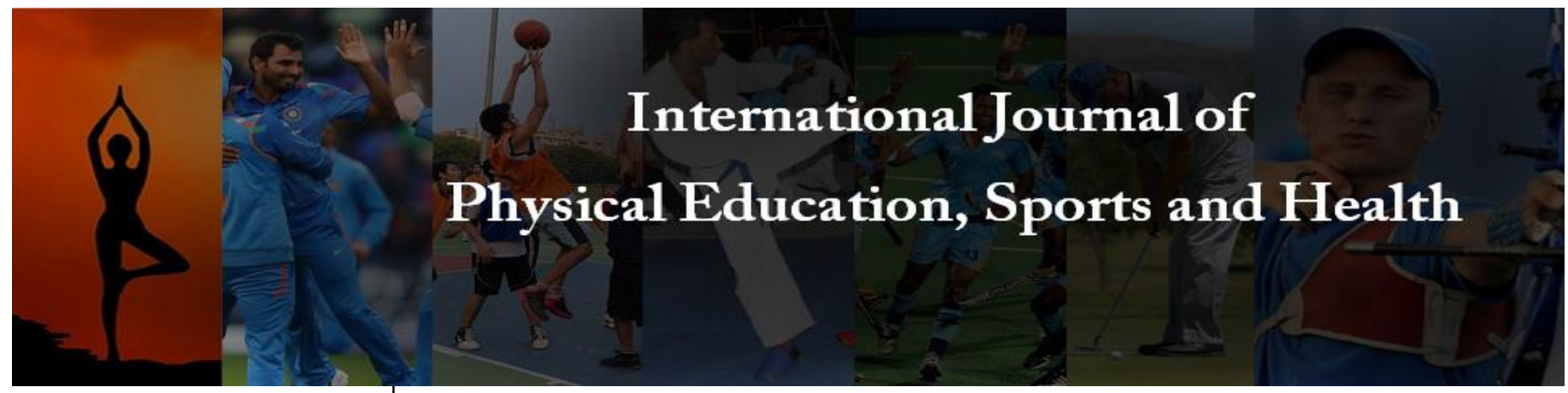

P-ISSN: 2394-1685

E-ISSN: 2394-1693

Impact Factor (ISRA): 5.38

IJPESH 2020; 7(6): 25-28

(C) 2020 IJPESH

www.kheljournal.com

Received: 16-09-2020

Accepted: 18-10-2020

A Chattopadhyay

Msc Sports Science RKMVERI,

Howrah, West Bengal, India
Corresponding Author:

A Chattopadhyay

Msc Sports Science RKMVERI,

Howrah, West Bengal, India

\section{Diabetes and athlete}

\section{A Chattopadhyay}

DOI: $\underline{\text { https://doi.org/10.22271/kheljournal.2020.v7.i6a.1893 }}$

\section{Abstract}

In this study, the main focus is on the athlete who suffers from Diabetes. We find that how over and under insulinization affects athletic performance. We also find how Diabetic athletes respond to different kind of exercise. Finally, we study the recovery process of Diabetic athletes.

Keywords: Diabetes, athlete, insulinization, performance, recovery

\section{Introduction}

Diabetes mellitus is the most usual category of metabolic diseases. It is distinguished by hyperglycemia occurring from defects in insulin secretion by the beta cell of the pancreas, insulin action, or both. Basically Diabetes is two types, one is Type-1 Diabetes and the other one is Type-2 Diabetes. Type-2 Diabetes is more common in people than Type-1. The Athlete also has Diabetes mellitus which may affect their performance in their respective field of sports. Each sport and the type of exercise have their consequence on diabetes dealing with many factors that notably affect glucose levels, including stress factor, level of water in the body, the rate of glycogenolysis and gluconeogenesis, and the secretion of counter-regulatory hormones. Diabetic athlete has a high risk of acute hypoglycemia or ketoacidosis and chronic complications including microvascular and macrovascular disease. Diabetes can't resist an Athlete to perform. One of the most magnificent of these athletes is Sir Steven Redgrave, winner of gold medals in rowing for Great Britain at five successive Olympic Games from 1984 to 2000 . He was diagnosed with diabetes mellitus about 2 years before the Sydney Olympic Games 2000.

\section{Defining the disease}

Type 1 Diabetes mellitus is distinguished by an autoimmune demolition of pancreatic beta cells that are the body erroneously destroys the tissues that make and secrete insulin. While insulin has many major functions in the body. The important functions of insulin during and after exercise are a) Stimulation of glucose uptake by the cells b) Restrict the glucose release from the liver c) inhibition of the release of fatty acids d) Enhance the protein synthesis in the body's cells e) increases the resynthesis of muscle glycogen after exercise.

Type 2 Diabetes mellitus is totally different from Type 1 Diabetes mellitus. In type 2 diabetes insulin is present adequately but it does not function properly to stimulate glucose uptake into cells. This condition is known as "Insulin resistance". The body tries to make up for this defect by secreting more and more amount of insulin, but eventually the reserve capacity of the pancreatic beta cells declines and the glucose concentration in the blood increases.

\section{The diabetic athlete}

Athletes with Diabetes who have achieved higher levels of performance have traditional patterns of carbohydrate feedings and insulinization that work for them. So individualized carbohydrate and insulin intake helps the athlete to maintain their blood glucose level in exercise condition and helps in optimum performance. For the most part, a lot of studies on the metabolic response to exercise in diabetic subjects used exercise protocols that did not reflect sports conditions. 
Most sports need short volumes of very high intensity effort, and most of what we know about exercise metabolism in diabetes is based on research that employed prolonged, moderate and constant-intensity exercise. Thus, for Athlete with diabetes, the metabolic demands and acute responses to participation in most sports are largely undisclosed.

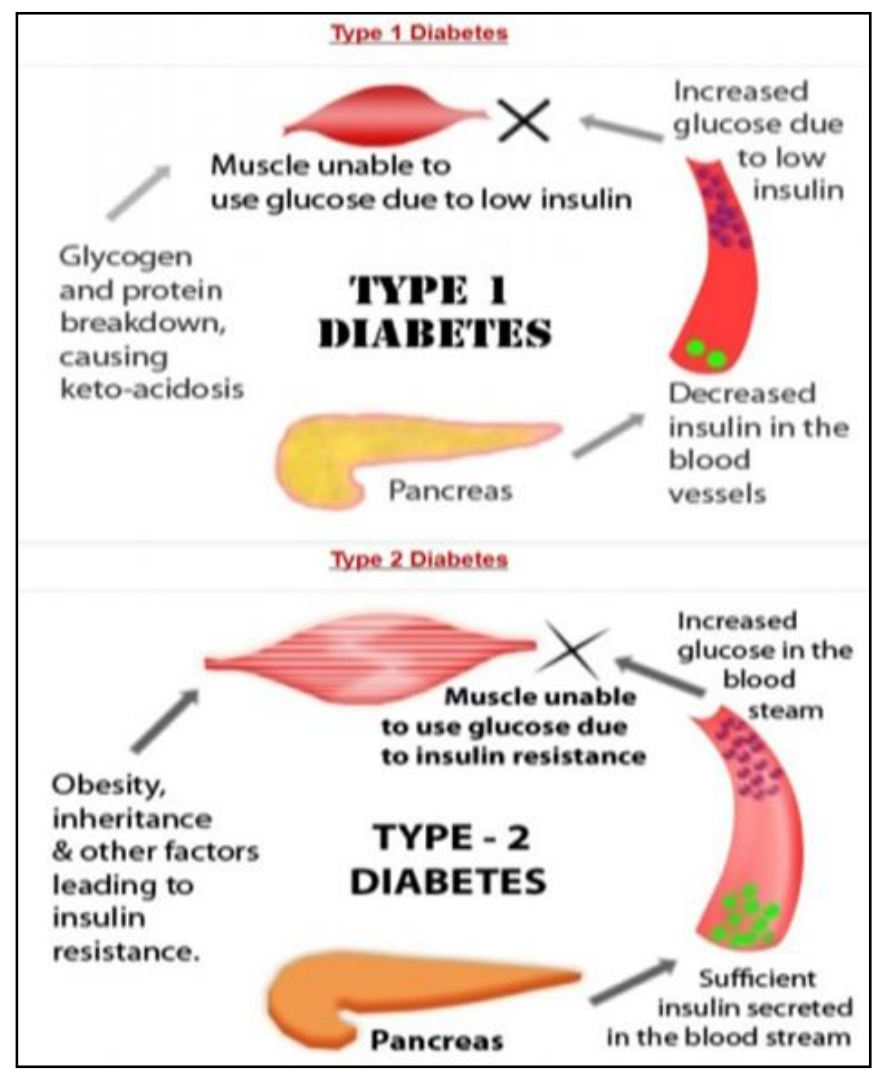

Fig 1: https://visual.ly/community/Infographics/health/diabetes-type1-diabetes-vs-type-2-diabetes

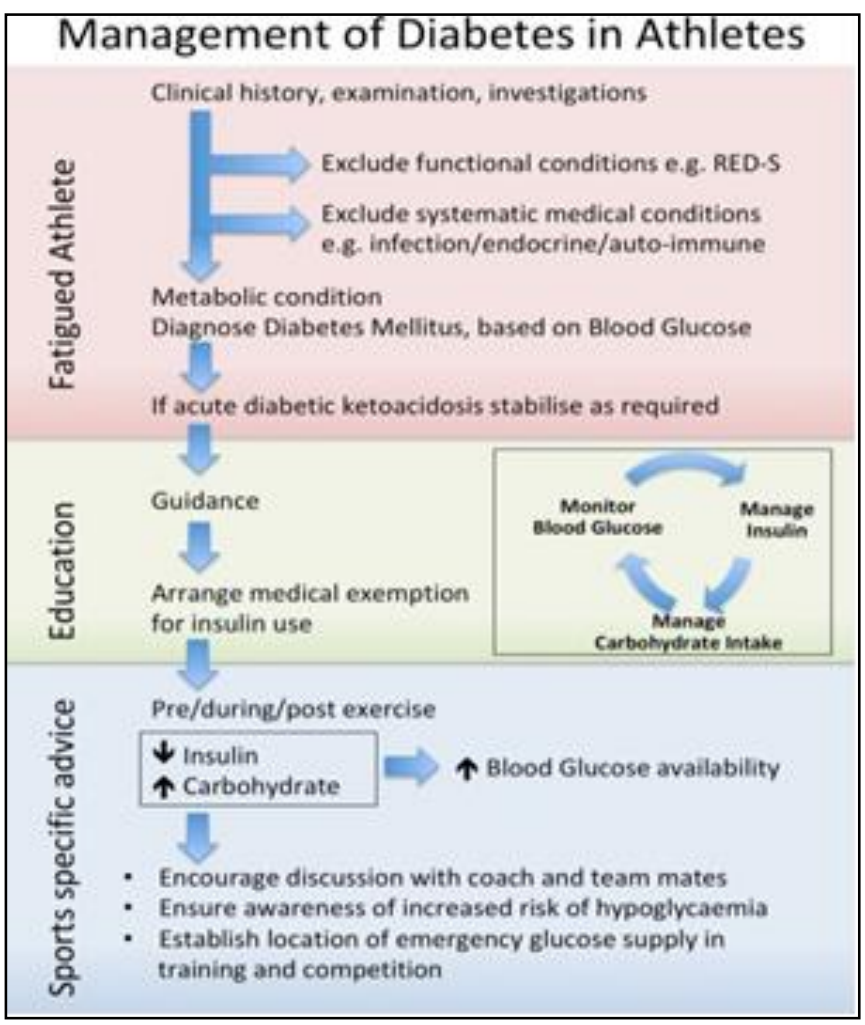

Fig 2: https://bjsm.bmj.com/content/early/2019/11/15/bjsports-2019101368

\section{Metabolic Responses to Acute Exercise}

In comparison to most hormones, concentrations of insulin in the blood reduce during exercise in Athletes without diabetes. It happens because less insulin is secreted from the pancreas and the contraction of skeletal muscle during exercise stimulates the Glucose uptake. Skeletal muscles are quantitatively the most prime tissue in the body for glucose uptake, especially in exercise conditions. Although insulin is boosting the Glucose uptake in resting cell. This decline in insulin secretion during exercise conditions at first appears contradictory. The natural decline in insulin concentration during exercise conditions is very much essential to prevent hypoglycemia ${ }^{[1]}$.

For people with Diabetes type 1 manage their blood glucose levels to take a pre-exercise insulin dose. The fuels used throughout the exercise condition are not distinctly different from fuels used by non-diabetic athletes, as long as the exercise intensity is average ${ }^{[2]}$.

The normal decline in blood insulin concentration during exercise condition in Athlete without diabetes and in Athlete with Type 2 Diabetes permits the two most chief fuels for exercise, carbohydrates, and fats, to be mobilized and used by the muscles. High concentrations of insulin in blood restrain the ability of the liver to release glucose and deliver it into the plasma (3). It also inhibits the release of fatty acids in the blood. So the athlete who takes insulin must be careful about its amount. Although, the normal decline in insulin concentration cannot happen in people with Type 1 Diabetes because they cannot produce it, and therefore a decrease in insulin production can't take place.

\section{Over- Insulinization and Under-Insulinization}

Athlete who has Diabetes takes insulin in most of the cases. So they have a high risk of insulin overdose or under dose.

Over-insulinization- The dispensation of too much insulin to control blood glucose. In the case of moderate exercise conditions, the most critical insulin target tissue is skeletal muscle, and muscle requires less or low insulin during the exercise phase. Accordingly, for brief, moderate-intensity exercise, a decrease of roughly $50 \%$ in the pre-exercise condition insulin dose is warranted in many cases ${ }^{[4,5]}$. If prolonged exercise takes place, that means the duration of the exercise is more than $90 \mathrm{~min}$, a $70-80 \%$ reduction of insulin may be needed to avoid a fall in blood glucose to a dangerous level that mean hypoglycemia. Over-insulinization can still happen because contractions make the muscle more sensitive to insulin. So the dose of insulin should be low before the exercise condition. The symptoms of Hypoglycemia are a lot of Sweating, pounding heart, shaking, confusion and drowsiness, difficulty with speech, in coordination, nausea vomiting, and headache. These are some common symptoms that help us to diagnosis the hypoglycemic condition.

Under-insulinization- It also can create a problem if the level of insulin is low in the blood. It can result in excessive concentrations of blood glucose which is called hyperglycemia. Very low insulin concentrations are insufficient to inhibit glucose release from the liver. A documented result of starting exercise with too little insulin concentration can cause hyperglycemia during exercise ${ }^{[6]}$. Additionally, other hormones of the body, especially glucagon secreted by the pancreas and epinephrine from the adrenal glands, become more effective in stimulating glucose production when insulin concentration is too low in the blood (7). The symptoms of Hyperglycemia are heavy thirst, Central fatigue, muscle cramps, blurred vision, nausea, 
drowsiness, and unbearable abdominal pain.

During low-intensity exercise or prolonged exercise conditions, the fatty acids become the major fuel source of energy for the active muscles. So Overinsulinization will inhibit fatty acid release from fat stores in the body, whereas underinsulinization will permit an immoderate mobilization of fatty acids, which can cause ketone production in the body and release by the liver, This condition called diabetic ketosis. The proper carbohydrate intake before any exercise can solve the insulin problem partially. Currently, we know very less about the metabolic adjustments and regulation to exercise in children athlete with diabetes. So we need to gain a proper idea about glucose management for both children and adults Athlete ${ }^{[8,9]}$.

\section{Resistance exercise for diabetic athlete}

Normally, Athlete's with Type 2 Diabetes who perform chronic resistance exercise, gain the same metabolic and muscle hypertrophy advantages as do nondiabetics athletes. But with the Athlete who has Type 1 Diabetes result is not the same. Type 1 Diabetes Athlete has higher systolic and diastolic blood pressures during bicycle ergometer and handgrip exercise compared to nondiabetic Athletes ${ }^{[10}$, ${ }^{11]}$. Massive exercise of all types, but mainly the resistance exercise, increases arterial pressure to very high levels in Athletes without diabetes. Such excessive pressures could damage the blood vessels in the eyes of those with diabetes. So the Athlete who has Type 1 Diabetes must be careful about resistance exercise. Athlete with diabetic autonomic neuropathies has difficulty controlling their blood pressure, cardiac output, and distribution of blood flow. As these components plays a major role during exercise condition, especially when such exercise is strenuous ${ }^{[12]}$.

\section{Recovery process in the diabetic athlete}

A proper recovery is very much needed for optimal performance. Some study suggests that diabetic athletes in soccer, swimming, and track and field may not recover as rapidly as Comparison with nondiabetic Athletes. This might be because the post exercise resynthesis of glycogen that means the storage form of glucose in the muscle and liver is slower in person with diabetes compared to others ${ }^{[13]}$. So the Athletes are unable to restore their glycogen level fully before the next practice or game session, which may affect their performance. Another problem is observed in the Diabetic athlete is "Delayed hypoglycemia". This phenomenon can occur for from 4 to 48 hours of after exercise. It can hamper the player's performance. It can disturb sleep, which could play a part to prolonged fatigue during the exercise phase ${ }^{[14]}$.

\section{Benefits of Regular Exercise for Diabetics}

Constant physical exercise now is acknowledged to have several benefits to health, not only for those Athlete who has diabetes, but for everyone. In addition to lowering the blood glucose level and increasing insulin sensitivity, regular physical exercise improves several of the recognized risk factors for cardiovascular disease. Serum cholesterol and triglyceride concentrations also decreases with physical activity, because of reduction in low density and very low density lipoproteins and increases in high density lipoprotein cholesterol. Also, moderate hypertension may improve. The resting pulse rate and cardiac work decrease in regular exercise; Psychological add ons of exercise, such as an growing sense of well-being, improved self-esteem, and an enhanced quality of life also may be important for those with either type I or type 2 diabetic athlete (15-17).

Hemoglobin A1c (HbA1C) is used as an indication of longterm blood glucose control, That is glucose levels that have existed for the previous $2-3$ months. If the value of $\mathrm{HbA} 1 \mathrm{c}$ is lower, it will be better. The fact that HbA1c is decreased by chronic exercise in people with Type2 Diabetes is very much important because this indicates that long-term glucose control has been realized and that with good control comes reduced risks for complications. Unfortunately, the same is not to be said for Type 1 Diabetes ${ }^{[18,19]}$.

\section{Conclusion}

Here we can see how diabetes hampers an athlete's performance. Although the Type 1 Diabetic athlete has more complication than Type 2 diabetic athlete. The response of diabetic athlete towards exercise is completely different. The insulin concentration plays a major role in their athletic career. They also need more time to recover properly. So the metabolic regulation of diabetic athlete and normal athlete is not same. Although there is a challenge but diabetic athlete can also perform well in their respective field.

\section{References}

1. A forty-year memoir of research on the regulation of glucose transport into muscle John O. Holloszy 01 MAR 2003https://doi.org/10.1152/ajpendo.00463 2002.

2. Raguso CA, AR Coggan, A Gastaldelli, LS Sidossis, EJ Bastyr III, RR Wolfe, et al. Lipid and carbohydrate metabolism in IDDM during moderate and intense exercise. Diabetes 1995;44:1066-1074

3. Wahren J. Glucose turnover during exercise in healthy man and in patients with diabetes mellitus. Diabetes 1979;28:82-88

4. Wasserman DH, SN Davis, et al. Fuel Metabolism during exercise in health and disease. In: N.B. Ruderman, J.T. Devlin, S.H. Schneider, and A. Kriska (eds.) Handbook of Exercise in Diabetes. Alexandria, VA: American Diabetes Association 2002, 66-99.

5. Schiffrin A, S Parikh. Accommodating planned exercise in type 1 diabetic patients on intensive treatment. Diabetes Care 1985;8:337-343

6. Berger M, P Berchtold, HJ Cuppers, H Drost, HK Kley, WA Muller, et al. Metabolic and hormonal effects of muscular exercise in juvenile type diabetics. Diabetologia 1977;13:355-365

7. Cryer PE. The prevention and correction of hypoglycemia. In: L.S. Jefferson and A.D. Cherrington (eds.) Handbook of Physiology, vol. 2. Oxford:Oxford University Press 2001, 1057-1093

8. Campaigne BN, TB Gilliam, ML Spencer, RM Lampman, MA Schork. Effects of a physical activity program on metabolic control and cardiovascular fitness in children with insulin-dependent diabetes mellitus. Diabetes Care 1984;7:57-62.

9. Dahl-Jørgensen K, HD Meen, KF Hanssen, O Aagenaes. The effect of excercise on diabetic control and hemoglobin A1 in children. Acta Paediatr. Scand 1980;283:53-56

10. Christensen NJ, H Galbo, A Gjerris, JH Henriksen, J Hilsted, M Kjaer, et al. Whole body and regional clearances of noradrenaline and adrenaline in man. Acta Physiol. Scand 1984;527:17-20.

11. Torffvit O, J Castenfors, U Bengtsson, CD Agardh. Exercise stimulation in insulin-dependent diabetics, normal increase in albuminuria with abnormal blood 
pressure response. Scand. J Lab. Invest 1987;47:253-259.

12. Hornsby G. Putting a lift in your workout. Diabetes Forecast 1990;43:55-60.

13. Hermansen, L. Resynthesis of muscle glycogen stores during recovery from prolonged exercsie in non-diabetic and diabetic subejcts. Acta Paediatr. Scand. 1980;283:3338.

14. MacDonald MJ. Postexercise late-onset hypoglycemia in insulin-dependent diabetic patients. Diabetes Care 1987; 10:584-588.

15. Bjorntorp P, de Jounge K, Sjostrom L, et al. The effect of physical training on insulin production in obesity. '11etabolism 1970;19:631-637.

16. Huttunen JK, Lansimies E, Voutilainen E, et al. Effect of moderate physical exercise on serum lipoprotein. Circulation 1979;60:1220-1229.

17. Kemmer FW, Berchtold P, Berger M, et al. Exerciseinduced fall of blood glucose in insulin-treated diabetics, unrelated to alteration of insulin mobilization. Diabetes 1979;28:1131-1137.

18. Laaksonen, DE, M Atalay, LK Niskanen, J Mustonen, CK Sen, TA Lakka, et al. Aerobic exercsie and the lipid profile in type 1 diabetic men: a randomized controlled trial. Med. Sci. Exerc. Sports 2000;32:1541-1548.

19. Landt KW, BN Campaigne, FW James, MA Sperling. Effects of exercise training on insulin sensitivity in adolescents with type I diabetes. Diabetes Care 1985;8:461-465. 